\title{
论 文拓扑流及其应用专题
}

\section{Skyrme模型中的拓扑结构}

\author{
邹丽平*，张鹏鸣* \\ 中国科学院近代物理研究所, 兰州 730000 \\ *联系人, 邹丽平, E-mail: zoulp@impcas.ac.cn; 张鹏鸣, E-mail: zhpm@impcas.ac.cn \\ 收稿日期: 2018-05-03; 接受日期: 2018-05-14; 网络出版日期: 2018-07-06 \\ 国家自然科学基金(批准号: 11575254, 11447105)资助
}

摘要 Skyrme模型具有丰富的拓扑结构, 包括重子数拓扑 $\pi_{3}\left(S^{3}\right)$ 、磁单极拓扑 $\pi_{2}\left(S^{2}\right)$ 和壳层拓扑(径向激 发量子数) $\pi_{1}\left(S^{1}\right)$ 等。首先讨论 $\mathrm{QCD}$ 与 Skyrme模型之间的关系, 并通过 $\mathrm{QCD}$ 中的磁单极拓扑性质进一步讨 论Skyrme模型中重子数拓扑分解问题. 此外还将具体求解Bogomol'nyi-Prasad-Sommerfield (BPS) Skyrme模 型中的具有不同磁单极拓扑数和壳层拓扑数的BPS skyrmion解.

关键词 Skyrme模型, skyrmion重子数拓扑, skyrmion磁单极拓扑, skyrmion壳层拓扑, BPS Skyrme模型

PACS: 03.75.Fi, 05.30.Jp, 67.40.Vs, 74.72.-h

\section{1 引言}

Skyrme模型是描述强相互作用的一个低能有 效模型, 被广泛应用于描述强子和原子核的相关性 质. 在该模型中最基本的物理场是 $\pi$ 场, 重子则表现 为模型中的拓扑孤立子(Skyrmion $)^{[1]}$. 在Skyrme提 出该模型之后, 就很快被用来描述気核和其他一些 轻核 ${ }^{[2,3]}$. 近年来, 由于计算机模拟的发展人们已经 能够用Skyrme模型构造许多原子核 ${ }^{[4-6]}$, 甚至是重 子数高达 108 的原子核 ${ }^{[7]}$. 因此理论上讲任意原子 核都可以通过Skyrme模型来构造.

Skyrme模型具有丰富的拓扑性质 ${ }^{[8,9]}$. 除 了skyrmion之外, 模型中还存在baby skyrmion、扭 结(Knot)等拓扑孤立子. 此外, 研究表明磁单极
拓扑也在Skyrme模型中起着重要作用 ${ }^{[10]}$. DuanGe-Cho规范势分解理论 ${ }^{[11-13]}$ 是研究规范场结构 性质的重要方法, 它能够分离出非阿贝尔规范场 的拓扑部分, 在规范场拓扑性质研究中有广泛应 用. 尤其是在研究量子色动力学(Quantum Chromodynamics, QCD)磁单极拓扑以及QCD真空的扭 结拓扑性质中有着重要应用 ${ }^{[14-16]}$. 通过Duan-GeCho规范势分解理论, QCD拉氏量可以与Skyrme模 型拉氏量紧密联系起来 ${ }^{[8]}$. 然而人们对Skyrme模 型中的磁单极物理少有讨论, 只是通过重子数拓 扑描述skyrmion. 磁单极是非微扰QCD中非常重 要的物理内容, 磁单极凝聚可以解释QCD中的色 禁闭现象. 鉴于QCD与Skyrme模型之间的关系, 磁 单极在Skyrme模型中也必然存在重要物理效应.

引用格式: 邹丽平, 张鹏鸣. Skyrme模型中的拓扑结构. 中国科学: 物理学 力学 天文学, 2018, 48: 100005

Zou L P, Zhang P M. The topological structures in Skyrme model (in Chinese). Sci Sin-Phys Mech Astron, 2018, 48: 100005, doi: 10.1360/SSPMA201800175 
文献 $[9,10]$ 提出Skyrme模型中的拓扑孤立子可以 通过磁单极(或者磁单极-反磁单极对)来理解, 其 中skyrmion可以理解为一个内嵌磁单极的拓扑孤立 子, baby skyrmion是磁单极-反磁单极对产生的涡旋, 而扭结则是由螺旋baby skyrmion在特定边界条件下 形成的稳定构型. 人们虽然不讨论Skyrme模型中的 磁单极拓扑, 然而在研究Skyrme模型非常重要的方 法rational map中就用到了具有磁单极拓扑的物理 场 $\hat{n}$. 最近在文献[10]中, 提出描述skyrmion的重子 数拓扑 $\pi_{3}\left(S^{3}\right)$ 可以进一步分解为磁单极拓扑以及径 向激发量子数, 分别描述 $\pi_{2}\left(S^{2}\right)$ 和 $\pi_{1}\left(S^{1}\right)$ 拓扑. 径向 激发量子数(壳层拓扑) 是该文提出的一种独立拓扑 数, 但最早由Manton等人 ${ }^{[17]}$ 在模拟多重子数原子核 时曾用来构造原子核的壳层结构. 在重子数拓扑 分解框架下, 由于系统能量的关系壳层量子数也被 认为是描述基态skyrmion的激发态量子数. 高激发 态skyrmion并不能稳定存在并最终会衰变到基态. 然而从拓扑上来讲, 由于磁单极和壳层拓扑数各自 满足不同的拓扑性质. 因此径向激发skyrmion的衰 变不会是随意的, 必将分别满足磁单极拓扑、壳层 拓扑数的守恒性质.

除了标准的Skyrme模型, Skyrme模型家族还有 许多推广的模型 ${ }^{[18]}$. 标准的Skyrme模型只考虑 $\pi$ 场 微分的二次方和四次方项, 但在推广的Skyrme模 型中可以根据一定的对称性原则添加其他高次 方项, 在文献[19]中讨论了包含有12次方项的推 广Skyrme模型动力学问题. 除此之外, Bogomol'nyiPrasad-Sommerfield (BPS) Skyrme模型是另一种非 常重要的推广Skyrme模型, 其拉氏量只包含了场微 分的0次方和 6 次方项. 标准的Skyrme模型解释原子 核时存在核子间束缚能偏大的问题 [20-22]. 而BPS模 型由于满足Bogomol'nyi条件, 因此其能量正比于 重子数 ${ }^{[23]}$, 属于零束缚能体系. 但在引入合适的库 仑相互作用和量子化修正等修正项, BPS模型可以 很好地解释原子核束缚能问题 ${ }^{[24]}$. 此外, 也可以 利用较小的二次方和四次方Skyrme项并作为BPS Skyrme模型的微扰项, 解决Skyrme模型束缚能过大 的问题 ${ }^{[25]}$. BPS Skyrme模型类似于核物理中的液滴 模型, 并成功用于描述重核、核物质以及中子星等 相关问题 ${ }^{[24,26]}$.
由于BPS Skyrme模型中skyrmion之间的结合能 为零, 因此其径向激发态也不存在能量过高的问题. 本文将讨论BPS skyrmion $\pi_{1}\left(S^{1}\right)$ 拓扑荷大于 1 的解, 通过一定的相互作用势, 我们可以得到精确的解析 解. 与常规BPS Skyrmion解不同的是, 在 $\pi_{1}\left(S^{1}\right)$ 拓扑 荷大于 1 时, 我们可以从能量密度以及重子数密度图 中看到skyrmion的壳层结构.

\section{2 从QCD到Skyrme模型}

在引言中我们提到Skyrme模型与 $\mathrm{QCD}$ 存在密 切关系. 实际上, QCD拉氏量通过一定的变换关系 可以得到Skyrme模型拉氏量. 首先, 让我们先回 顾Duan-Ge-Cho 规范势分解理论 ${ }^{[11,13]}$. 引入内部空 间常矢量 $\hat{\mathbf{n}}$, 满足条件

$\hat{D}_{\mu} \hat{\mathbf{n}}=\partial_{\mu} \hat{\mathbf{n}}+g \hat{A}_{\mu} \times \hat{\mathbf{n}}=0$.

由此可以解得

$\hat{A}_{\mu}=A_{\mu} \hat{\mathbf{n}}+\mathbf{C}_{\mu}, \quad \mathbf{C}_{\mu}=-\frac{1}{g} \hat{\mathbf{n}} \times \partial_{\mu} \hat{\mathbf{n}}$.

$\hat{A}_{\mu}$ 也被称为限制规范势 (Restricted Potential), 满足完 整的 $\mathrm{SU}(2)$ 规范变换:

$\delta \hat{A}_{\mu}=\frac{1}{g} \hat{D}_{\mu} \boldsymbol{\alpha}, \delta \hat{\mathbf{n}}=-\boldsymbol{\alpha} \times \hat{\mathbf{n}}$,

并且保留了非阿贝尔规范场所有的拓扑性质. 其 中场 $\hat{\mathbf{n}}$ 描述了同伦映射 $\pi_{2}\left(S^{2}\right)$, 因此, $\hat{A}_{\mu}$ 具有非阿贝 尔规范场的磁单极拓扑, 其中 $\mathbf{C}_{\mu}$ 即为磁单极势. 磁 单极在QCD的色禁闭问题中扮演了重要角色, 对 偶超导模型告诉我们磁单极凝聚形成的QCD真空 束缚了所有带色电荷的粒子, 因此QCD禁闭势来 源于 $\mathrm{QCD}$ 胶子场中的限制规范场. 而这一结论也 得到了 $\mathrm{QCD}$ 格点计算的确认 ${ }^{[27]}$. 但由于条件(1), $\hat{A}$ 只能够描述 $\mathrm{QCD}$ 胶子场的低能部分动力学. 因此, 在Duan-Ge-Cho分解理论下要表述完整的QCD胶子 场 $\mathbf{A}_{\mu}$ 还需要引入色场 $\mathbf{X}_{\mu}$ :

$\mathbf{A}_{\mu}=\hat{A}_{\mu}+\mathbf{X}_{\mu}=A_{\mu} \hat{\mathbf{n}}-\frac{1}{g} \hat{\mathbf{n}} \times \partial_{\mu} \hat{\mathbf{n}}+\mathbf{X}_{\mu}$,

其中, $\hat{A}_{\mu}$ 也被称为色中性限制胶子场, $\mathbf{X}_{\mu}$ 被称为价 胶子场(Valence Potential). 理论认为限制胶子场不 
能够作为组分存在于核子中, 而价胶子场可以作为 组分存在于胶球混杂态等强子态中 ${ }^{[28]}$.

由Duan-Ge-Cho分解, 可以得到

$$
\begin{aligned}
\mathbf{F}_{\mu \nu} & =\partial_{\mu} \mathbf{A}_{v}-\partial_{v} \mathbf{A}_{\mu}+g \mathbf{A}_{\mu} \times \mathbf{A}_{v} \\
& =\hat{F}_{\mu \nu}+\hat{D}_{\mu} \mathbf{X}_{v}-\hat{D}_{v} \mathbf{X}_{\mu}+g \mathbf{X}_{\mu} \times \mathbf{X}_{v},
\end{aligned}
$$

并且QCD拉氏量可以表示为

$$
\begin{aligned}
\mathcal{L}= & -\frac{1}{4} \hat{F}_{\mu \nu}-\frac{1}{4}\left(\hat{D}_{\mu} \mathbf{X}_{v}-\hat{D}_{v} \mathbf{X}_{\mu}\right)^{2} \\
& -\frac{g}{2} \hat{F}_{\mu v} \cdot\left(\mathbf{X}_{\mu} \times \mathbf{X}_{v}\right)-\frac{g^{2}}{4}\left(\mathbf{X}_{\mu} \times \mathbf{X}_{v}\right)^{2} .
\end{aligned}
$$

让我们回到Skyrme模型, 其拉氏量由标量 场 $\omega$ 和描述 $\pi$ 介子的矢量场合构成. 进一步可以表 示为

$$
\begin{aligned}
\mathcal{L}= & \frac{\mu^{2}}{4} \operatorname{tr} L_{\mu}^{2}+\frac{\alpha}{32} \operatorname{tr}\left(\left[L_{\mu}, L_{\nu}\right]\right)^{2} \\
= & -\frac{\mu^{2}}{4}\left[\frac{1}{2}\left(\partial_{\mu} \omega\right)^{2}+2 \sin ^{2} \frac{\omega}{2}\left(\partial_{\mu} \hat{\mathbf{n}}\right)^{2}\right] \\
& -\frac{\alpha}{16}\left[\sin ^{2} \frac{\omega}{2}\left(\partial_{\mu} \omega \partial_{\nu} \hat{\mathbf{n}}-\partial_{\nu} \omega \partial_{\mu} \hat{\mathbf{n}}\right)^{2}\right. \\
& \left.+4 \sin ^{4} \frac{\omega}{2}\left(\partial_{\mu} \hat{\mathbf{n}} \times \partial_{\nu} \hat{\mathbf{n}}\right)^{2}\right],
\end{aligned}
$$

其中, $\mu$ 和 $\alpha$ 是相互作用常数, 并且

$$
\begin{aligned}
& L_{\mu}=U \partial_{\mu} U^{\dagger}, \\
& U=\exp \left(\frac{\omega}{2 \mathrm{i}} \boldsymbol{\sigma} \cdot \hat{\mathbf{n}}\right)=\cos \frac{\omega}{2}-\mathrm{i}(\boldsymbol{\sigma} \cdot \hat{\mathbf{n}}) \sin \frac{\omega}{2} .
\end{aligned}
$$

有意思的是仅仅通过引入条件

$\omega(r)=\pi$,

Skyrme拉氏量可以进一步简化到Skyrme-Faddeev模 型:

$\mathcal{L} \rightarrow-\frac{\mu^{2}}{2}\left(\partial_{\mu} \hat{\mathbf{n}}\right)^{2}-\frac{\alpha}{4}\left(\partial_{\mu} \hat{\mathbf{n}} \times \partial_{\nu} \hat{\mathbf{n}}\right)^{2}$.

Skyrme-Faddeev模型在物理中有重要应用, 被认为 是QCD低能有效模型. 该模型中研究较多的是baby skyrmion和扭结拓扑孤立子. 也有许多理论研究用 以解释胶球, 胶球可以通过有限能量的扭结拓扑孤 立子来理解.
现在让我们来比较QCD拉氏量(7)与Skyrme模 型拉氏量(8)和(11). 在QCD中, 考虑真空凝聚后限制 规范场 $\hat{A}_{\mu}$ 将获得质量, 其拉氏量可以进一步表示为

$$
\begin{aligned}
\mathcal{L}= & -\frac{\mu^{2}}{2} \hat{A}_{\mu}-\frac{1}{4} \hat{F}_{\mu \nu}-\frac{1}{4}\left(\hat{D}_{\mu} \mathbf{X}_{v}-\hat{D}_{v} \mathbf{X}_{\mu}\right)^{2} \\
& -\frac{g}{2} \hat{F}_{\mu \nu} \cdot\left(\mathbf{X}_{\mu} \times \mathbf{X}_{v}\right)-\frac{g^{2}}{4}\left(\mathbf{X}_{\mu} \times \mathbf{X}_{v}\right)^{2} .
\end{aligned}
$$

当不考虑价场 $\mathbf{X}_{\mu}$ 时, 并且把场 $\mathbf{A}_{\mu}$ 积分掉以后得到的 有效场即为

$\mathcal{L}=-\frac{1}{4 g^{2}}\left(\partial_{\mu} \hat{\mathbf{n}} \times \partial_{v} \hat{\mathbf{n}}\right)^{2}-\frac{\mu^{2}}{2}\left(\partial_{\mu} \hat{\mathbf{n}}\right)^{2}$.

此即Skyrme-Faddeev模型拉氏量(11). 因此QCD 在Duan-Ge-Cho规范势分解理论下可以严格得 到Skyrme-Faddeev模型. 不仅如此, QCD拉氏量还 可以等价地表示为完整的Skyrme模型拉氏量(8). 为 此, 我们可以将价场 $\mathbf{X}_{\mu}$ 简单表示为

$\mathbf{X}_{\mu}=f_{1} \partial_{\mu} \hat{\mathbf{n}}+f_{2} \hat{\mathbf{n}} \times \partial_{\mu} \hat{\mathbf{n}}, \phi=f_{1}+\mathrm{i} f_{2}$.

当满足 $\partial_{\mu} \phi=0, A_{\mu}=\partial_{\mu} \omega$ 条件时, $\mathrm{QCD}$ 拉氏量简 化为

$$
\begin{aligned}
\mathcal{L}= & -\frac{1}{4 g^{2}}\left(1-g^{2} \phi^{*} \phi\right)^{2}\left(\partial_{\mu} \hat{\mathbf{n}} \times \partial_{\nu} \hat{\mathbf{n}}\right)^{2}-\frac{1}{4} \phi^{*} \phi\left(\partial_{\mu} \omega \partial_{\nu} \hat{\mathbf{n}}\right. \\
& \left.-\partial_{\mu} \omega \partial_{\nu} \hat{\mathbf{n}}\right)^{2}-\frac{\mu^{2}}{2}\left[\left(\partial_{\mu} \omega\right)^{2}+\frac{1}{g^{2}}\left(\partial_{\mu} \hat{\mathbf{n}}\right)^{2}\right] .
\end{aligned}
$$

得到了一个与Skyrme模型拉氏量基本一致的QCD 低能有效拉氏量. 式(11)和(15)告诉我们Skyrme模型 实际上是一个磁单极场与标量场 $\omega$ 相互作用的 QCD 有效模型. 这意味着磁单极是Skyrme模型的基本物 理之一, Skyrme模型中的baby skyrmion、扭结以及 重子数拓扑也可以通过磁单极拓扑来解释.

在引言中我们提到本文将讨论Skyrme模型中 新的拓扑解, 尤其是求解Skyrme模型中的 $\pi_{1}\left(S^{1}\right)$ 拓 扑径向激发态解. 然而在标准的Skyrme模型该径向 激发态解的能量过高, 不能够用来解释高重子数的 基态原子核, 因此我们将进一步讨论BPS Skyrme模 型中的相关问题. BPS Skyrme模型拉氏量与式(8)不 同, 它是推广的Skyrme模型, 由场微分的6次方项 和 0 次方势能项组成. 其拉氏量表示为

$\mathcal{L}_{\mathrm{BPS}}=\mathcal{L}_{6}+\mathcal{L}_{0}=\frac{\lambda^{2}}{24}\left[\operatorname{tr}\left(\epsilon^{\mu \nu \rho \sigma} U^{\dagger} \partial_{\mu} U U^{\dagger} \partial_{\nu} U U^{\dagger} \partial_{\rho} U\right)\right]^{2}$ 


$$
-\mu^{2} V\left(U^{\dagger} U\right)
$$

事实上在推广的Skyrme模型中, 其拉氏量中可以有 更高次方的微分项, 这些微分项可能与QCD也有存 在着密切关系. 这是因为QCD有效拉氏量可以表示 为 ${ }^{[29]}$

$$
\begin{aligned}
\mathcal{L}_{\mathrm{eff}}= & -\frac{1}{4}\left(D_{v}^{a b} F_{\rho \mu}^{b} D_{v}^{a c} F_{\rho \mu}^{c}+D_{\mu}^{a b} F_{\mu \nu}^{b} D_{\rho}^{a c} F_{\rho \nu}^{c}\right) \\
& -\frac{1}{12} \operatorname{tr}\left[C_{1} F^{2}+\frac{4}{3} C_{2} F^{4}-\frac{16}{9} C_{3} F^{6}\right],
\end{aligned}
$$

存在 $F_{\mu \nu}$ 的高次方项.

\section{Skyrme模型中的 $\pi_{2}\left(S^{2}\right)$ 磁单极拓扑以 及 $\pi_{1}\left(S^{1}\right)$ 壳层拓扑}

严格求解Skyrme模型是很困难的, 尤其是在重 子数大于 1 的原子核体系, 其空间构型不再具有球 对称性. 因此人们也通常采用一定的方法针对不 同的原子核引入相应的试探函数, rational map是求 解高重子数Skyrme模型通常采用的方法. 在该方法 中, 不同重子数的skyrmion具有不同的pion场 $\hat{n}$. 但 从 QCD与Skyrme模型的关系中我们知道场 $\mathbf{n}$ 是可以 表征 $\pi_{2}\left(S^{2}\right)$ 拓扑的, 即磁单极拓扑. Rational map提供 了一种求解Skyrme模型的方法, 但磁单极拓扑并不 依赖于rational map具体形式. 通过场合我们可以得 到磁单极拓扑荷:

$$
\begin{aligned}
M & =\frac{ \pm 1}{8 \pi} \int \epsilon_{i j k} H_{i j} \mathrm{~d} \sigma_{k} \\
& =\frac{ \pm 1}{8 \pi} \int \epsilon_{i j k} \hat{\mathbf{n}} \cdot\left(\partial_{i} \hat{\mathbf{n}} \times \partial_{j} \hat{\mathbf{n}}\right) \mathrm{d} \sigma_{k} .
\end{aligned}
$$

Skyrme模型中存在特殊的磁单极解, 即

$\omega(r)=\pi, \hat{\mathbf{n}}=\hat{r}$,

其中, $\hat{r}$ 是球坐标系中径向单位矢量. 可以验证该磁 单极解具有磁荷

$$
\begin{aligned}
M & = \pm \frac{1}{8 \pi} \int \epsilon_{i j k} \hat{\mathbf{n}} \cdot\left(\partial_{i} \hat{\mathbf{n}} \times \partial_{j} \hat{\mathbf{n}}\right) \mathrm{d} \sigma_{k} \\
& = \pm \frac{1}{8 \pi} \int \epsilon_{i j k} \hat{r} \cdot\left(\partial_{i} \hat{r} \times \partial_{j} \hat{r}\right) \mathrm{d} \sigma_{k}= \pm 1 .
\end{aligned}
$$

当然, 磁单极解是发散的, 系统能量不收玫. 因此一 般情况下要求标量场 $\omega(r) \neq 0$, 这时候Skyrmion的重 子数:

$$
\begin{aligned}
B & =\frac{1}{24 \pi^{2}} \int \epsilon_{i j k} \operatorname{Tr}\left(L_{i} L_{j} L_{k}\right) \mathrm{d} x^{3} \\
& =\frac{1}{8 \pi^{2}} \int \epsilon_{i j k} \partial_{i} \omega \hat{\mathbf{n}} \cdot\left(\partial_{i} \hat{\mathbf{n}} \times \partial_{j} \hat{\mathbf{n}}\right) \sin ^{2} \frac{\omega}{2} \mathrm{~d} r^{3} \\
& =\frac{1}{\pi} \int \sin ^{2} \frac{\omega}{2} \mathrm{~d} \omega .
\end{aligned}
$$

我们可以看到这里重子数 $B$ 依赖于函数 $\omega$ 的边界条 件. 在以往的工作中, 人们通常取边界条件:

$\omega(0)=2 \pi, \omega(\infty)=0$.

从而满足重子数 $B=1$. 从这里我们也可以看 出重子数与磁单极拓扑数在数学形式上并不 相等, 但它们都描述了 skyrmion的某些拓扑性质. 并且skyrmion也可以由磁单极解理解, 在 $\omega$ 取不 同的边界条件时, 比如 $\omega(0)=2 \pi, \omega(\infty)=\pi$ 或 者 $\omega(0)=\pi, \omega(\infty)=0$ 可以得到重子数为 $1 / 2$ 能 量发散的skyrmioin解, 分别在空间无穷远处以及原 点处包含了一个磁单极, 这也是为什么它们的能量 是发散的. 这些结果告诉我们, 常规的skyrmion解实 际上内嵌有磁单极, 磁单极场 $\hat{\mathbf{n}}$ 与标量场 $\omega(r)$ 的相互 作用消除了磁单极带来的发散性质.

另一方面, 当场 $\omega(r)$ 在零点处的边界条件取 为 $\omega(0)=2 n \pi$, 这就使得式(21)中的重子数为 $B=n$. 通过求解, 此时系统的能量为 ${ }^{[10]}$

$E_{n} \simeq \frac{n(n+1)}{2} E_{1}$.

因此在物理上, 该边界条件给出的结果不能很好地 用来解释原子核. 原子核中由于核子间结合能的存 在, skyrmion能量需要满足要求:

$E_{B}<B \cdot E_{1}$

因此, $\omega(0)=2 n \pi$ 解可以认为是基态skyrmion的激发 态, 这也是我们称呼 $U(1)$ 参数 $n$ 为径向激发量子数的 原因.

前面我们只讨论了单位磁单极荷情况下的 重子数在 $\omega(r)$ 场不同边界条件下的情况, 事实上 
我们也可以推广到任意磁单极荷 $M=m$. 这主 要是因为 $S^{3} \simeq S^{2} \times S^{1}$, 因此重子数拓扑 $\pi_{3}\left(S^{3}\right)$ 可 以进一步分解为 $\pi_{2}\left(S^{2}\right)$ 和 $\pi_{1}\left(S^{1}\right)$, 分别描述了场 $\hat{\mathbf{n}}$ 的 磁单极拓扑和 $\omega(r)$ 场的壳层拓扑. 具体可以表示 为 $\pi_{3}\left(S^{3}\right) \simeq \pi_{2}\left(S_{2}\right) \times \pi_{1}\left(S^{1}\right)$, 重子数为

$$
\begin{aligned}
B & =\frac{1}{8 \pi^{2}} \int \epsilon_{i j k} \partial_{i} \omega \hat{\mathbf{n}} \cdot\left(\partial_{i} \hat{\mathbf{n}} \times \partial_{j} \hat{\mathbf{n}}\right) \sin ^{2} \frac{\omega}{2} \mathrm{~d} r^{3} \\
& =\frac{1}{8 \pi^{2}} \int \sin ^{2} \frac{\omega}{2} \frac{\mathrm{d} \omega}{\mathrm{d} x^{i}} \epsilon_{i j k} \hat{\mathbf{n}} \cdot\left(\partial_{i} \hat{\mathbf{n}} \times \partial_{j} \hat{\mathbf{n}}\right) \mathrm{d} x^{j} \mathrm{~d} x^{k} \\
& =\frac{1}{4 \pi^{2}} \int \sin ^{2} \frac{\omega}{2} \mathrm{~d} \omega \int \hat{\mathbf{n}} \cdot(\mathrm{d} \hat{\mathbf{n}} \wedge \mathrm{d} \hat{\mathbf{n}})=m n,
\end{aligned}
$$

其中, $\omega=\omega\left(x^{i}\right), \hat{\mathbf{n}}=\hat{\mathbf{n}}\left(x^{j}, x^{k}\right)$, 磁单极荷为 $m$, 壳层拓 扑数为 $n$. 当然这里 $B=m n$ 的skyrmion可以是不稳定 的, 在一定程度上可以认为是高重子数原子核的激 发态, 它们可以衰变到 $n=1, B=m$ 的基态. 但是由 于拓扑性质的存在, 这些skyrmion的衰变也必须满 足拓扑荷守恒的性质, 即磁单极荷和壳层拓扑荷必 须分别守恒. 对于前面我们提到的BPS Skyrme模型, 由于Bogomol'nyi条件的存在系统束缚能为零, 能量 满足 $E_{B}=B E_{1}$. 因此可以认为 $\mathrm{BPS}$ 模型中壳层拓扑 数大于 1 的解是稳定的.

\section{BPS Skyrme 模型中的 $n>1$ 的解}

让我们回到BPS Skyrme模型拉氏量(16), 在此 引入测地投影 ${ }^{[23]}$ :

$$
\begin{aligned}
& U=\mathrm{e}^{\mathrm{i} \hat{\xi} \hat{\mathbf{n}} \cdot \vec{\tau}}, \\
& \hat{\mathbf{n}}=\frac{1}{1+|u|^{2}}\left(\begin{array}{c}
u+\bar{u} \\
-\mathrm{i}(u-\bar{u}) \\
|u|^{2}-1
\end{array}\right),
\end{aligned}
$$

其中, $\hat{\mathbf{n}}$ 为磁单极场, $\xi=\frac{\omega}{2}$. 由此可以得到运动方程:

$$
\begin{aligned}
& \frac{\lambda^{2} \sin ^{4} \xi}{\left(1+|u|^{2}\right)^{4}} \partial_{\mu}\left(\sin ^{2} \xi H^{\mu}\right)-\mu^{2} V_{\xi}=0, \\
& \partial_{\mu}\left(\frac{K^{\mu}}{\left(1+|u|^{2}\right)^{2}}\right)=0,
\end{aligned}
$$

其中, $V_{\xi}=\partial_{\xi} V$, 并且

$$
\begin{aligned}
H_{\mu} & =\frac{\partial\left(\epsilon^{\alpha \nu \rho \sigma} \xi_{\nu} u_{\rho} \bar{u}_{\sigma}\right)^{2}}{\partial \xi^{\mu}}, \\
K_{\mu} & =\frac{\partial\left(\epsilon^{\alpha \nu \rho \sigma} \xi_{\nu} u_{\rho} \bar{u}_{\sigma}\right)^{2}}{\partial \bar{u}^{\mu}} .
\end{aligned}
$$

在球对称条件下, 函数满足

$\xi=\xi(r), \quad u(\theta, \phi)=g(\theta) \mathrm{e}^{(\mathrm{i} m \phi)}$.

关于函数 $u$ 的运动方程可以简化为

$$
\frac{1}{\sin \theta} \partial_{\theta}\left(\frac{g^{2} g_{\theta}}{\left(1+g^{2}\right)^{2} \sin \theta}\right)-\frac{g g_{\theta}^{2}}{\left(1+g^{2}\right)^{2} \sin ^{2} \theta}=0 .
$$

方程存在解析解:

$g(\theta)=\tan \left(\frac{\theta}{2}\right)$.

此时磁单极场即为我们前面讨论的简单情况 $\hat{\mathbf{n}}=\hat{r}$, 可以看出式(29)中常数 $m$ 即为磁单极荷. 此外标量 场 $\xi(r)$ 的运动方程为

$$
\frac{m^{2} \lambda^{2} \sin ^{2} \xi}{2 r^{2}} \partial_{r}\left(\frac{\sin ^{2} \xi \xi_{r}}{r^{2}}\right)-\mu^{2} V_{\xi}=0 .
$$

该方程可以进一步简化, 在一定的相互作用势的条 件下, 方程存在 compacton解 ${ }^{[23,24]}$.

同时由于BPS Skyrme模型可以满足Bogomol'ny 条件, 因此存在一阶微分方程. 根据式(26), 我们可以得到BPS Skyrme模型的能量积分函数

$$
\begin{aligned}
E= & \int \mathrm{d} x^{3}\left(\frac{\lambda^{2} \sin ^{4} \xi}{\left(1+|u|^{2}\right)^{4}}\left(\epsilon^{m n l} \mathrm{i} \xi_{m} u_{n} \bar{u}_{l}\right)^{2}+\mu^{2} V\right) \\
= & \int \mathrm{d} x^{3}\left(\frac{\lambda \sin ^{2} \xi}{\left(1+|u|^{2}\right)^{2}}\left(\epsilon^{m n l} \mathrm{i} \xi_{m} u_{n} \bar{u}_{l}\right) \pm \mu \sqrt{V}\right)^{2} \\
& \mp \int \mathrm{d} x^{3} \frac{2 \mu \lambda \sin ^{2} \xi \sqrt{V}}{\left(1+|u|^{2}\right)^{2}}\left(\epsilon^{m n l} \mathrm{i} \xi_{m} u_{n} \bar{u}_{l}\right) \\
\geqslant & \mp \int \mathrm{d} x^{3} \frac{2 \mu \lambda \sin ^{2} \xi \sqrt{V}}{\left(1+|u|^{2}\right)^{2}}\left(\epsilon^{m n l} \mathrm{i} \xi_{m} u_{n} \bar{u}_{l}\right) \\
= & \pm\left(2 \lambda \mu \pi^{2}\right)\left[\frac{-\mathrm{i}}{\pi^{2}} \int \mathrm{d} x^{3} \frac{\sin ^{2} \xi \sqrt{V}}{\left(1+|u|^{2}\right)^{2}}\left(\epsilon^{m n l} \mathrm{i} \xi_{m} u_{n} \bar{u}_{l}\right)\right] \\
= & 2 \lambda \mu \pi^{2} C_{1} B,
\end{aligned}
$$

其中, $B$ 为重子数, $C_{1}$ 为积分常数. 在Bogomol'nyi条 件下, 能量最低的解满足一阶微分BPS方程:

$$
\frac{\lambda \sin ^{2} \xi}{\left.\left(1+|u|^{2}\right)^{2}\right)}\left(\epsilon^{m n l} \mathrm{i} \xi_{m} u_{n} \bar{u}_{l}\right)=\mp \mu \sqrt{V} .
$$

通过条件(29), 方程(34)可以简化为

$$
\frac{m \lambda}{2 r^{2}} \sin ^{2} \xi(r) \xi^{\prime}(r)=\mp \mu \sqrt{V} .
$$


因此比较式(32)和(35), 可以发现运动方程(32)实际 上是BPS方程(34)的平方形式. 通过无量纲化 $r \rightarrow$ $\sqrt[3]{\frac{\mu}{\lambda}} r$, 方程可以简化为

$\int m \frac{\sin ^{2} \xi}{\sqrt{V}(\xi)} \mathrm{d} \xi=\int 2 r^{2} \mathrm{~d} r$

通过选取一定的相互作用势 $V(\xi)$, 可以严格求解上 述方程. 根据拓扑边界条件, 通常取为

$V=\frac{1}{2} \operatorname{Tr}(1-U)=1-\cos \xi$.

在此条件下可以得到解为

$\cos ^{3} \frac{\xi}{2}= \pm \frac{\sqrt{2}}{4 n} r^{3}+c_{0}$,

其中, $c_{0}$ 是积分常数, 并满足 $c_{0}=\cos ^{3} \frac{\xi(r=0)}{2}$. 由此, 重 子数可以表示为

$$
\begin{aligned}
B & =\frac{2 m}{\pi} \int \sin ^{2} \xi \xi^{\prime} \mathrm{d} r=\frac{2 m}{\pi} \int \mathcal{B} \mathrm{d} r \\
& =\left.\frac{m}{\pi}\left(\xi-\frac{1}{2} \sin (2 \xi)\right)\right|_{\xi(0)} ^{\xi(\infty)} .
\end{aligned}
$$

在边界条件

$\xi(0)=n \pi, \quad \xi(\infty)=0$,

重子数表示为 $B=m n$, 其中 $m$ 为磁单极拓扑数, $n$ 为 壳层拓扑数. 同时根据Bogomol'nyi条件, 系统能量 可以表示为

$$
\begin{aligned}
E & =4 \pi \lambda \mu \int \mathcal{E} \mathrm{d} r=4 \pi \lambda \mu \int\left(\frac{n^{2} \sin ^{4} \xi}{4 r^{4}}+V\right) r^{2} \mathrm{~d} r \\
& =8 \pi \lambda \mu \int V r^{2} \mathrm{~d} r .
\end{aligned}
$$

由于三角函数的特殊性质, 这里我们只讨论 $n=$ 1,2 情况下的解. 当 $n=1$, 我们有

$\xi(r)= \begin{cases}2 \arccos \sqrt[3]{\frac{\sqrt{2}}{4 n} r^{3}}, & r \in[0, \sqrt{2} \sqrt[3]{m}], \\ 0, & r \geqslant \sqrt{2} \sqrt[3]{m} .\end{cases}$

相应的能量为

$E=\frac{64 \sqrt{2}}{15} \pi m \mu \lambda$.
图 1中的曲线分别是函数 $\xi$, 相应BPS skyrmion的 能量密度E以及重子数密度 $\mathcal{B}$ 的曲线, $\mathcal{E}, \mathcal{B}$ 分别由 式(41)和(39)定义.

当 $n=2$, 我们有

$\xi(r)= \begin{cases}2 \arccos \sqrt[3]{\frac{\sqrt{2}}{4 n} r^{3}-1}, & r \in[0, \sqrt{2} \sqrt[3]{2 m}], \\ 0, & r \geqslant \sqrt{2} \sqrt[3]{2 m} .\end{cases}$

相应的能量为 $E=\frac{128 \sqrt{2}}{15} \pi n \mu \lambda$, 解的具体情况如图 2 .

从图 2中我们可以看出壳层拓扑数 $n=2$ 的解 在壳层 $n=1$ 对应的位置处能量密度和重子数密 度曲线有很大的变化. 进一步可以分析壳层拓扑 数 $n=2$ 的解在坐标 $r=\sqrt{2} \sqrt[3]{m}$ 处的性质, 该空间坐 标对应于壳层数 $n=1$ BPS skyrmion 解的右边界. 不 难发现从原点到该处系统的能量积分值为

$E_{21}=8 \pi \lambda \mu \int_{0}^{\sqrt{2} \sqrt[3]{m}} V(r) r^{2} \mathrm{~d} r=\frac{64 \sqrt{2}}{15} \pi m \mu \lambda$.

这刚好对应于壳层拓扑 $n=1$ 的skyrmion解的总能 量. 同时从 $r=\sqrt{2} \sqrt[3]{m}$ 到边界的 $r=\sqrt{2} \sqrt[3]{2 m}$ 系统所具 有的能量为

$E_{22}=\frac{64 \sqrt{2}}{15} \pi m \mu \lambda$.

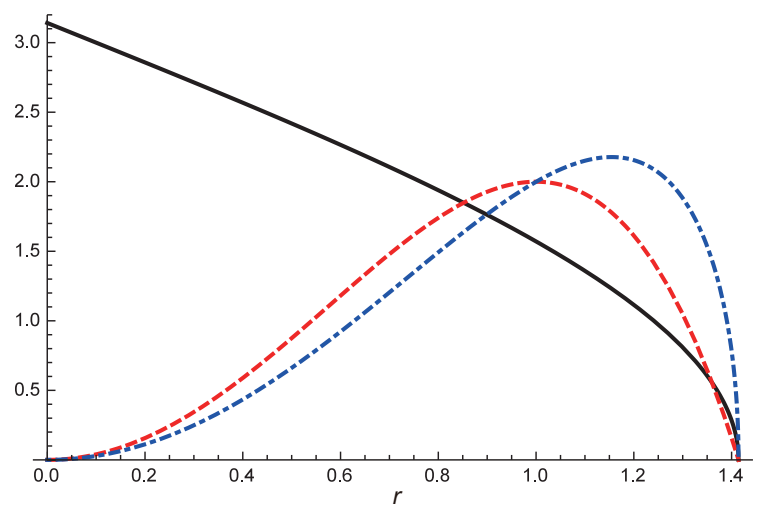

图 1 (网络版彩图) 磁单极数 $m=1$ 壳层数 $n=1$ 的BPS skyrmion解, 其中实线对应于标量场 $\xi$, 虚线为能量密度 $\mathcal{E}$, 点虚线为重子数密度 $\mathcal{B}$. 图中横坐标为 $r$, 其单位为 $\sqrt[3]{u / \lambda}$

Figure 1 (Color online) The BPS skyrmion solution with monopole number $m=1$ and shell number $n=1$, the solid line, dashed line and dotdashed line correspond to the solutions of scalar field $\xi$, energy density $\mathcal{E}$ and baryon charge density $\mathcal{B}$, respectively. The dimensionless abscissa $r$ is in the unite of $\sqrt[3]{u / \lambda}$. 


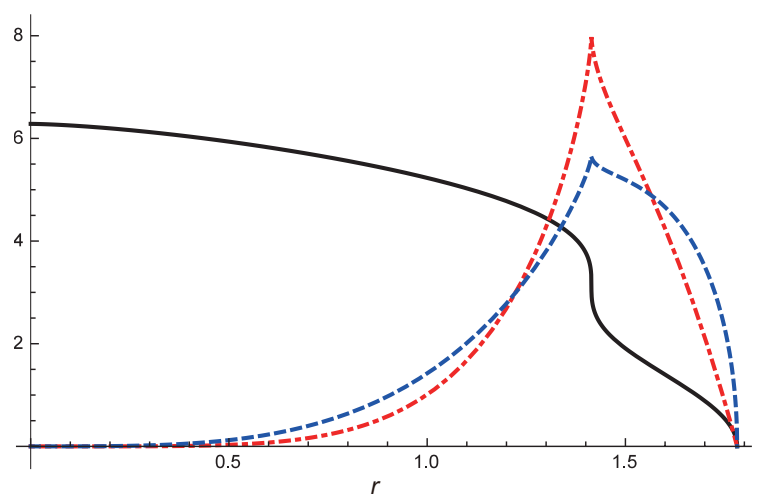

图 2 (网络版彩图) 磁单极数 $m=1$ 壳层数 $n=2$ 的BPS skyrmion解, 其中实线对应于标量场 $\xi$, 虚线为能量密度 $\mathcal{E}$, 点虚线为重子数密度 $\mathcal{B}$. 图中横坐标为 $r$, 其单位为 $\sqrt[3]{u / \lambda}$

Figure 2 (Color online) The BPS skyrmion solution with monopole number $m=1$ and shell number $n=2$, the solid line, dashed line and dotdashed line correspond to the solutions of scalar field $\xi$, energy density $\mathcal{E}$ and baryon charge density $\mathcal{B}$, respectively. The dimensionless abscissa $r$ is in the unite of $\sqrt[3]{u / \lambda}$.

这说明对于 $n=2$ 的解, 存在两个壳层结构. 在每一 个壳层上分别具有重子数 $B=m$ 的核子, 这与核物 理中壳层模型具有相似的性质.

\section{5 结论}

Skyrme模型是强相互作用的低能有效模型, 在 核物理中该模型的解skyrmion可以用来构造原子 核. 从QCD出发研究了磁单极在Skyrme模型中的物 理意义, 分析了磁单极拓扑与skyrmion重子数拓扑 之间的关系, 进一步阐述了重子数拓扑可以分解为 磁单极拓扑数和壳层拓扑数. 由于空间 $S^{3}$ 可以近似 的表示为 $S^{3} \simeq S^{2} \times S^{1}$, 因此描述重子数拓扑的同 伦映射 $\pi_{3}\left(S^{3}\right)$ 可以分解为 $\pi_{2}\left(S^{2}\right) \times \pi_{1}\left(S^{1}\right)$, 这说明重 子数拓扑具有内在的结构. Duan-Ge-Cho规范场分 解理论告诉我们Skyrme模型实际上是 $\mathrm{QCD}$ 的一个 等效模型. 因此我们可以从QCD出发理解Skyrme模 型中的一些物理问题. 通常人们认为Skyrmion只具 有 $\pi_{3}\left(S^{3}\right)$ 重子数拓扑, 然而在 $\mathrm{QCD}$ 中最基本的拓扑
孤立子却是磁单极. 磁单极在 $\mathrm{QCD}$ 起着极为重要的 角色, 色禁闭现象可以通过磁单极凝聚形成QCD真 空来解释; 而Skyrme模型是QCD低能有效模型, 因 此磁单极子在Skyrme模型中也应该有重要的物理 内容. 然而磁单极拓扑 $\pi_{2}\left(S^{2}\right)$ 在Skyrme模型中少有 讨论, 这主要是由于决定 skyrmion拓扑性质的场 $U$ 只 能定义 $\pi_{3}\left(S^{3}\right)$ 拓扑. 从Dauan-Ge-Cho规范势分解理 论出发, 场合描述规范场的磁单极拓扑性质. 根 据QCD与Skyrme模型的关系我们认为skyrmion也应 该具有内在的磁单极拓扑. 事实上通过磁单极子, 我们可以更好地理解Skyrme模型中的许多物理. 比 如baby skyrmion可以认为是有磁单极-反磁单极对 形成的浴旋, 扭结可以认为是螺旋磁单极-反磁单极 浴旋环形成的稳定拓扑孤立子, 而skyrmion则可以 认为是内嵌的磁单极子与标量场 $\omega$ 相互作用形成的 有限能量孤立子解.

通过重子数拓扑分解可以进一步研究Skyrme 模型中新的物理解. 在标准的Skyrme模型中对 于壳层拓扑数 $n>1$ 的解, skyrmion 能量具有关 系 $E_{n} \simeq n(n+1) E_{1} / 2$. 这并不满足原子核中核子 间束缚能的要求, 即 $E_{n}<n E_{1}$, 因此对于 $n>1$ 的 解skyrmion也被认为是激发态. 激发态可以衰变到 基态, 但是根据拓扑荷守恒性质, 这些skyrmion的衰 变并不是任意的, 必须满足磁单极拓扑荷和壳层 拓扑荷守恒关系. 鉴于束缚能的问题, 我们进一步 研究了BPS Skyrme模型中的壳层拓扑荷大于 1 的解. BPS模型由于满足Bogomol'nyi条件, 因此是无束缚 能系统, skyrmion的能量正比于重子数. 在本文中我 们求解了壳层数 $n=1,2$, 磁单极拓扑数为 $m$ 重子数 为 $B=m n$ 的BPS skyrmion解. 在 $n=2$ 的解中我们确 实发现了系统可以通过两个壳层来理解, 并且每个 壳层具有 $m$ 个重子, 能量相同. 这与核物理中的壳模 型类似, 当然也不完全等同. 我们也希望进一步通 过这些性质讨论原子核、核物质以及中子星的相关 物理.

\section{参考文献}

1 Skyrme T H R. A non-linear field theory. Proc R Soc A-Math Phys Eng Sci, 1961, 260: 127-138; Particle states of a quantized meson field. Proc 
R Soc A-Math Phys Eng Sci, 1961, 262: 237-245; A unified field theory of mesons and baryons. Nucl Phys, 1962, 31: 556-569

2 Verbaarschot J J M, Walhout T S, Wambach J, et al. Symmetry and quantization of the two-skyrmion system: The case of the deuteron. Nucl Phys A, 1987, 468: 520-538

3 Braaten E, Carson L. Deuteron as a toroidal Skyrmion. Phys Rev D, 1988, 38: 3525-3539

4 Houghton C J, Manton N S, Sutcliffe P M. Rational maps, monopoles and skyrmions. Nucl Phys B, 1998, 510: 507-537

5 Battye R A, Sutcliffe P M. Solitonic fullerene structures in light atomic nuclei. Phys Rev Lett, 2001, 86: 3989-3992; Skyrmions, fullerenes and rational maps. Rev Math Phys, 2002, 14: 29-85

6 Piette B M A G, Probert G I. Towards skyrmion stars: Large baryon configurations in the Einstein-Skyrme model. Phys Rev D, 2007, 75: 125023

7 Feist D T J, Lau P H C, Manton N S. Skyrmions up to baryon number 108. Phys Rev D, 2013, 87: 085034

8 Cho Y M. Monopoles and knots in Skyrme theory. Phys Rev Lett, 2001, 87: 252001

9 Cho Y M, Park B S, Zhang P M. New interpretation of Skyrme theory. Int J Mod Phys A, 2008, 23: 267-298

10 Cho Y M, Kimm K, Yoon J H, et al. New topological structures of Skyrme theory: Baryon number and monopole number. Eur Phys J C, 2017, 77: 88

11 Cho Y M. Restricted gauge theory. Phys Rev D, 1980, 21: 1080-1088

12 Cho Y M. Glueball spectrum in extended quantum chromodynamics. Phys Rev Lett, 1981, 46: 302-306

13 Duan Y S, Ge M L. SU(2) gauge theory and electrodynamics with $N$ magnetic monopoles (in Chinese). Sci Sin, 1979, 9: 1072-1081 [段一士, 葛 墨林. SU(2)规范理论与 $N$ 个磁单极运动体系的电动力学. 中国科学, 1979, 9: 1072-1081]

14 Cho Y M. Colored monopoles. Phys Rev Lett, 1980, 44: 1115-1118

15 Zou L P, Pak D G, Zhang P M. Monopoles without magnetic charges: Finite energy monopole-antimonopole configurations in $\mathrm{CP}^{1}$ model and restricted QCD. Phys Lett B, 2014, 728: 650-657

16 Zou L P, Zhang P M, Pak D G. Knot topology in QCD. Phys Lett B, 2013, 726: 436-443

17 Manton N S, Piette B M A G. Understanding Skyrmions using rational maps. In: Casacuberta C, Miró-Roig R M, Verdera J, et al., eds. European Congress of Mathematics. Progress in Mathematics, vol 201. Basel: Birkhäuser, 2001

18 Marleau L. The Skyrme model and higher order terms. Phys Lett B, 1990, 235: 141-146

19 Gudnason S B, Nitta M. A higher-order Skyrme model. J High Energ Phys, 2017, 2017: 28

20 Battye R A, Sutcliffe P M. Symmetric skyrmions. Phys Rev Lett, 1997, 79: 363-366

21 Battye R A, Sutcliffe P M. Skyrmions and the pion mass. Nucl Phys B, 2005, 705: 384-400

22 Battye R A, Sutcliffe P M. Skyrmions with massive pions. Phys Rev C, 2006, 73: 055205

23 Adam C, Sánchez-Guillén J, Wereszczyński A. BPS Skyrme model and baryons at large $\mathrm{N}_{c}$. Phys Rev D, 2010, 82: 085015

24 Adam C, Naya C, Sanchez-Guillen J, et al. Bogomol'nyi-Prasad-Sommerfield Skyrme model and nuclear binding energies. Phys Rev Lett, 2013, 111: 232501

25 Bonenfant E, Marleau L. Nuclei as near BPS Skyrmions. Phys Rev D, 2010, 82: 054023

26 Adam C, Naya C, Sanchez-Guillen J, et al. BPS Skyrmions as neutron stars. Phys Lett B, 2015, 742: 136-142

27 Cundy N, Cho Y M, Lee W, et al. The static quark potential from the gauge invariant Abelian decomposition. Phys Lett B, 2014, 729: 192-198

28 Cho Y M, Pham X Y, Zhang P M, et al. Glueball physics in QCD. Phys Rev D, 2015, 91: 114020

29 Nedelko S N, Voronin V E. Domain wall network as QCD vacuum and the chromomagnetic trap formation under extreme conditions. Eur Phys J A, 2015, 51: 45 


\title{
The topological structures in Skyrme model
}

\author{
ZOU LiPing* \& ZHANG PengMing* \\ Institute of Modern Physics, Chinese Academy of Sciences, Lanzhou 730000, China
}

\begin{abstract}
In this paper, we study the new topological structures in Skyrme model. Besides the baryon topology $\pi_{3}\left(S_{3}\right)$, monopole topology $\pi_{2}\left(S^{2}\right)$ and shell topology (radial number) $\pi_{1}\left(S^{1}\right)$ are also investigated. Skyrme model is an effective model of quantum choromodynamics (QCD) at low energy, which could be understood from QCD lagrangian with Duan-Ge-Cho decomposition theory. With this and the the monopole topology in QCD, we discuss the decomposition of baryon topology in standard Skyrme model and Bogomol'nyi-Prasad-Sommerfield (BPS) Skyrme model. New BPS skyrmion solutions are shown with different monopole topology and shell topology.
\end{abstract}

Skyrme model, skyrmion baryon topology, skyrmion monopole topology, skyrmion shell topology, BPS Skyrme model

PACS: 03.75.Fi, 05.30.Jp, 67.40.Vs, 74.72.-h

doi: $10.1360 /$ SSPMA2018-00175 